\title{
La Implementación del Aprendizaje Cooperativo de ELE en Serbia ${ }^{1}$
}

\author{
Andjelka Pejović ${ }^{2}$ \\ Universidad de Belgrado \\ MAJA ANDRIJEVIĆ 3 \\ Universidad John Nesbitt
}

Recibido: 30 abril 2017 / Aceptado: 27 julio 2017

ISSN: $1697-7467$

\begin{abstract}
RESUMEN: El presente trabajo propone investigar si los profesores de ELE en Serbia siguen los preceptos de las nuevas tendencias en la didáctica de lenguas extranjeras y si aplican en clase los conocimientos adquiridos en seminarios de formación, incluidas las técnicas del aprendizaje interactivo. Para ello se ha elaborado un cuestionario de un total de 14 preguntas, al que han contestado 11 profesores de ELE, y se ha procedido a un análisis cualitativo de las respuestas. El análisis muestra que los profesores de ELE serbios recurren al aprendizaje cooperativo en sus clases, aunque no siempre sean conscientes de ello. Asimismo, muestran un evidente afán de motivar a los alumnos y capacitarlos para ser autónomos a largo plazo. Se concluye que los seminarios de formación de profesores dan resultados positivos y que entre los temas de futuros seminarios debería figurar también la evaluación de los métodos del aprendizaje cooperativo.
\end{abstract}

Palabras clave: aprendizaje cooperativo, aprendizaje interactivo, diferencias individuales, actitudes epistemológicas, ELE.

The Implementation of Cooperative Learning of Spanish as a Foreign Language in Serbia

\begin{abstract}
This paper is an intention to survey if teachers of Spanish as a foreign language in Serbia follow the precepts of the new trends in FLT and if they apply in class the knowledge acquired in training seminars, including the techniques of interactive learning. For this purpose a questionnaire containing 14 questions has been elaborated. Eleven teachers of Spanish as a foreign language participated in the research, and then we carried out a qualitative analysis of their answers. The analysis shows that Serbian teachers do use the cooperative learning strategies in their classes, although they are not always aware of it. The results also reveal an evident eagerness to motivate students and enable them to be autonomous as a long-term goal. Additionally, the investigation shows that the teacher training seminars give positive results; therefore, the evaluation of cooperative learning methods should also be included among possible future seminar topics.
\end{abstract}

\footnotetext{
${ }^{1}$ Este trabajo se enmarca en el proyecto de investigación número178014, financiado por el Ministerio de Educación Ciencia y Desarrollo Tecnológico de la República de Serbia.

${ }^{2}$ andjelka.pejovic@fil.bg.ac.rs

${ }^{3}$ mandrijevic@naisbitt.edu.rs
} 
Keywords: collaborative learning, interactive learning, individual differences, epistemological attitudes, Spanish as a foreign language

El alumno es quien debe construir el aprendizaje, el maestro solo le acompaña en el camino (Lev Vygotsky)

\section{INTRODUCCIÓN}

Los cambios en el mundo globalizado a principios del siglo XXI muestran la necesidad de desarrollar la educación humanista y la emancipación personal. La clave de dicha educación es la identificación de las necesidades de los alumnos, para que estos sean independientes, asuman las responsabilidades y participen activamente en el proceso de aprendizaje, donde el profesor tampoco debe olvidarse de autoactualizarse (Mikanović 2015). «Para que el alumno se emancipe, es necesario que el profesor se olvide por completo del autoritarismo y medidas represivas y que no considere a los alumnos meros objetos en la enseñanza» (Branković \& Mikanović, 2011: 583). Los fundamentos de la educación humanista y emancipadora surgen de los preceptos del constructivismo, cuyo paradigma se centra en el proceso mismo de la adquisición y no en el resultado final. El quid de este proceso es la construcción del significado, o sea, que el alumno aprenda a aprender (mientras aprende) y se convierta en un sujeto activo en clase (Freire, 1998).

Ya en la segunda mitad de los años sesenta empezó a llamar la atención el aprendizaje interactivo, llamado aprendizaje colaborativo (collaborative learning), pero actualmente el tema sigue vigente. Una de las principales razones inevitablemente son las nuevas tecnologías y su uso en clase, en la enseñanza en general y en el aprendizaje de las lenguas extranjeras en particular. Es un hecho que los alumnos hoy día tienen la competencia informática lo suficientemente desarrollada como para poder explotarla en clase de lengua extranjera. Más aún, no excluimos la posibilidad de que algunos de estos alumnos estén mejor alfabetizados informáticamente que sus profesores, lo que habría que ver como una ventaja en cuanto al aprendizaje autónomo de los alumnos de lengua extranjera y sobre todo como una fuente de motivación sumamente importante.

Teniendo en cuenta que en la República de Serbia se organizan seminarios de formación de profesores de lenguas extranjeras de temas muy variados, como por ejemplo el desarrollo de la competencia comunicativa, los nuevos métodos en la enseñanza de lenguas extranjeras, el uso de las nuevas tecnologías, la motivación, los materiales auténticos en la enseñanza, etc. (Jovanović \& Pejović, 2012: 411), el principal objetivo del presente trabajo consiste en comprobar si los profesores de ELE en Serbia siguen los preceptos de las nuevas tendencias en la didáctica de lenguas extranjeras y si aplican en clase los conocimientos adquiridos, entre ellos las técnicas del aprendizaje interactivo, o sea, cooperativo. 


\section{ESTUdios PRECEDENTES}

\subsection{Aprendizaje cooperativo / colaborativo ${ }^{4}$}

Los primeros estudios sobre el aprendizaje colaborativo datan de mediados de los años sesenta $^{5}$ y principios de los setenta, como podemos constatar a partir de numerosos artículos de investigación, y están marcados por distintos enfoques y acercamientos a la cuestión de una enseñanza lo más eficaz posible. Se basan en los estudios de los psicólogos Jean Piaget y Lev Vygotsky, quienes destacan la pertinencia de la interacción social en el aprendizaje (Richards \& Rodgers, 2009: 191), por lo que el aprendizaje colaborativo se enmarca en la línea del enfoque comunicativo. Johnson, Johnson \& Stanne (2000: 1) hablan del aprendizaje colaborativo, que para ellos «es uno de los ámbitos más extraordinarios y fértiles de la teoría, la investigación y la práctica en la educación». Los autores señalan que este tipo de instrucción está muy bien integrada en los programas educativos en general debido a varios factores. En primer lugar, el aprendizaje interactivo está basado en distintas teorías ya confirmadas en la antropología, sociología, economía, psicología, ciencias políticas etc. (Johnson, Johnson \& Stanne, 2000: 2). En segundo lugar, este procedimiento se ha mostrado muy útil, aplicable y eficaz, puesto que da numerosos resultados positivos: mayores logros y aciertos, un raciocinio más elevado, una mejor retención de conocimientos, aumento de la motivación, desarrollo social y cognitivo, reducción de estereotipos y prejuicios, apreciación de las diferencias, etc. Finalmente, toda una gama de métodos de aprendizaje colaborativo apuestan por este tipo de instrucción. Los diez más conocidos o más referidos se presentan en la siguiente tabla: ${ }^{6}$

Tabla 1. Métodos modernos de aprendizaje colaborativo

\begin{tabular}{|c|c|c|}
\hline Johnson \& Johnson & Mid 1960s & $\begin{array}{c}\text { Learning Together \& } \\
\text { Alone }\end{array}$ \\
\hline DeVries \& Edwards & Early 1970s & $\begin{array}{c}\text { Teams-Games- } \\
\text { Tournaments (TGT) }\end{array}$ \\
\hline
\end{tabular}

\footnotetext{
${ }^{4}$ Panitz distingue entre aprendizaje colaborativo y aprendizaje cooperativo. Según el autor, «collaboration is a philosophy of interaction and personal lifestyle where individuals are responsible for their actions, including learning and respect the abilities and contributions of their peers; cooperation is a structure of interaction designed to facilitate the accomplishment of a specific end product or goal through people working together in groups» (Panitz, 1997: 3). Para Dillenbourg (1999), en el proceso cooperativo los participantes dividen el trabajo, resuelven subtareas individualmente y luego construyen resultados parciales en el output final. En la enseñanza colaborativa, los individuos hacen el trabajo juntos, o sea, construyen juntos el significado. En este trabajo usamos indistintamente lexemas ‘colaboración' y ‘cooperación' a pesar de que optamos por el término aprendizaje cooperativo, que parece prevalecer en español.

${ }^{5}$ A finales de los años sesenta Spencer Kagan desarrolló las llamadas estructuras cooperativas (Trujillo Sáez, 2002: 155).

${ }^{6}$ Los autores subrayan que la diferencia de métodos no supone su contradicción, por lo que todos pueden usarse en una misma clase, en distintas asignaturas por ejemplo (Johnson, Johnson \& Stanne, 2000: 14).
} 


\begin{tabular}{|c|c|c|}
\hline Sharan \& Sharan & Mid 1970s & Group Investigation \\
\hline Johnson \& Johnson & Mid 1970s & Constructive Controversy \\
\hline Aronson \& Associates & Late 1970s & Jigsaw Procedure \\
\hline Slavin \& Associates & Late 1970s & $\begin{array}{c}\text { Student Teams } \\
\text { Achievement Divisions } \\
\text { (STAD) }\end{array}$ \\
\hline Cohen & Early 1980s & Complex Instruction \\
\hline Slavin \& Associates & Early 1980s & $\begin{array}{c}\text { Team Accelerated } \\
\text { Instruction (TAI) }\end{array}$ \\
\hline Kagan & Mid 1980s & $\begin{array}{c}\text { Cooperative Learning } \\
\text { Structures }\end{array}$ \\
\hline $\begin{array}{c}\text { Stevens, Slavin, \& } \\
\text { Associates }\end{array}$ & Late 1980s & $\begin{array}{c}\text { Cooperative Integrated } \\
\text { Reading \& Composition } \\
\text { (CIRC) }\end{array}$ \\
\hline
\end{tabular}

Fuente: Johnson, Johnson \& Stanne, 2000: 3-4

Por tanto, el aprendizaje colaborativo es el término genérico referente a esta serie de métodos que se utilizan en clase con fines educativos (Johnson, Johnson \& Stanne, 2000: 3). Para poder elegir el adecuado, es necesario tener en cuenta lo siguiente (Sharan 2014: 803):

- ¿Qué método, modelo o procedimiento es el más apropiado para el contenido que ha de aprenderse?

- ¿Qué destrezas sociales y de aprendizaje se requieren?

- ¿Es apropiado el modelo para la edad de los alumnos?

- ¿Están preparados los alumnos para asumir la responsabilidad por su aprendizaje?

- ¿Está preparado el profesor para dar indicaciones necesarias?

- ¿De cuánto tiempo se dispone para la implementación del modelo adecuado?

- ¿Cómo se han formado los grupos?

- ¿Cuál es el tamaño óptimo del grupo?

- ¿Hay elementos que puedan estar en conflicto con normas culturales locales?

- ¿Qué tipo de premio (si existe) se recomienda, individual o a nivel de grupo?

Además, este tipo de aprendizaje no es «trabajo espontáneo en grupo, sin más preparación por parte del profesor y de los estudiantes» (Trujillo Sáez \& Ariza Pérez, 2006: 12), sino que, como señala Carrió Pastor (2007: 2), es un aprendizaje basado en cooperación, trabajo en equipo, comunicación y responsabilidad, donde cada miembro tiene un determinado papel del que se hace responsable y cada uno aporta valor al resultado final, que evalúan todos los participantes. 


\subsection{Aprendizaje cooperativo en la enseñanza de lenguas extranjeras}

Siendo la competencia comunicativa el principal objetivo de la enseñanza de lenguas extranjeras, y teniendo en cuenta que la comunicación supone interacción, que es el hilo del aprendizaje cooperativo, no extraña que el enfoque basado en interacción y colaboración encuentre un lugar destacado en este campo. Según Richards \& Rodgers (2009: 190), los objetivos en la enseñanza de idiomas desde esta perspectiva son los siguientes:

- Dar oportunidades para una adquisición naturalista de la segunda lengua mediante el uso de actividades en parejas y en grupo.

- Proporcionar a los profesores una metodología que les permita alcanzar este objetivo y que se pueda aplicar a diversos entornos curriculares.

- Hacer posible prestar atención prioritaria a unidades léxicas, estructuras lingüísticas y funciones comunicativas concretas a través del uso de tareas interactivas.

- Dar a los alumnos oportunidades de desarrollar unas estrategias de aprendizaje y comunicación que tengan buenos resultados.

- Aumentar la motivación de los alumnos, reducir su estrés y crear un clima afectivo positivo en el aula.

McGroarty (1989, apud Richards \& Rodgers, 2009: 192) señala las siguientes ventajas del enfoque interactivo en la enseñanza de lenguas extranjeras:

- Una mayor frecuencia y variedad en la práctica de la segunda lengua por medio de diferentes tipos de interacción.

- La posibilidad de desarrollar o usar la lengua de una manera que contribuya al desarrollo cognitivo y al aumento de las destrezas lingüísticas.

- Unas oportunidades de integrar la lengua en la instrucción basada en el contenido.

- Unas oportunidades de incluir una mayor variedad de materiales curriculares a fin de estimular el aprendizaje del idioma y el conceptual.

- La libertad que se da a los profesores para dominar nuevas habilidades docentes, sobre todo las que hacen hincapié en la comunicación.

- Las oportunidades de que los alumnos actúen a modo de recursos los unos para los otros, asumiendo así un papel más activo en su aprendizaje.

Al aprendizaje cooperativo se puede recurrir para la enseñanza de diversos contenidos (de gramática, pronunciación, vocabulario, etc.), igual que se pueden usar distintos tipos de actividades (Richards \& Rodgers, 2009: 192-193). Lo importante es que el alumno tenga un papel activo y que interactúe y colabore con los demás alumnos, mientras que el profesor sirve de facilitador del aprendizaje. 


\section{Metodología}

\subsection{Instrumento de la investigación}

Como instrumento de la investigación se ha elaborado un cuestionario, en serbio, que contiene 14 preguntas: cinco son de tipo general (se refieren a la edad, el sexo, la afiliación, la experiencia docente y la experiencia laboral (docente) previa), y nueve se refieren al proceso docente. La mayoría de las preguntas son abiertas ${ }^{7}$, primero porque no hemos querido influir en las respuestas de los profesores dándoles posibles pistas, y segundo, porque hemos querido obtener datos cualitativos, al saber de antemano que el número de los participantes no iba a ser elevado, debido a que el español todavía no está muy presente en el sistema educativo en la República de Serbia. De hecho, según las investigaciones realizadas, el español se enseña en una veintena de escuelas primarias y alrededor de una decena de escuelas secundarias. ${ }^{8}$ Sin embargo, debido al número insuficiente de horas lectivas, algunos profesores trabajan tanto en primaria como en secundaria, por lo que el número de profesores de español que trabajan en escuelas no es muy alto. ${ }^{9}$ Lo demuestra también el número de los participantes en los seminarios de formación de profesores, que gira en torno a 25 , aunque no todos trabajan en la enseñanza (por ejemplo, son estudiantes o licenciados en filología hispánica interesados en la enseñanza y en la formación continua). Por todo ello, las preguntas incluidas en el cuestionario se han elegido teniendo en cuenta, por un lado, las investigaciones anteriores que se han realizado en Serbia, y por otro, los contenidos de los seminarios de formación de profesores de ELE, que se organizan en el mismo país, para ver el seguimiento de la formación continua de los profesores y de su labor docente, desde el punto de vista de las nuevas tendencias en la didáctica de lenguas extranjeras. El cuestionario, enviado por correo electrónico, se realizó entre el 1 y el 20 de abril de 2017, de forma anónima, a través de la plataforma Google forms. ${ }^{10}$

\subsection{Participantes}

El cuestionario fue enviado a más de 20 profesores, de varias escuelas y ciudades, y respondido por 11 personas (9 profesoras y 2 profesores). Su edad varía entre 29 y 48 años (uno de los encuestados no ha revelado su edad) y su experiencia docente oscila entre 2 y 17 años. Seis profesores trabajan solo en primaria y dos solo en secundaria, mientras que dos participantes trabajan tanto en primaria como en secundaria. Uno de los participantes

\footnotetext{
${ }^{7}$ En el caso de las preguntas cerradas hemos pedido de los participantes la ejemplificación, con lo que prácticamente las nueve preguntas son abiertas.

${ }^{8}$ Según una investigación realizada por Jovanović y Sánchez Radulović (2013), en 2010 había 21 escuelas primarias y 13 escuelas secundarias en las que se enseñaba el español, y en el año escolar 2011/2012 el español como lengua optativa-obligatoria se enseñaba en 27 escuelas primarias y 6 escuelas secundarias. No obstante, según datos no oficiales de los que disponemos, este número ha bajado en los últimos años.

${ }^{9}$ Más información acerca del estatus del español en el sistema educativo serbio puede encontrarse en: Pejović y Jovanović 2012, Jovanović y Sánchez Radulović 2013, Jovanović y Pejović 2013.

${ }^{10}$ Hemos optado por esta forma debido a que es una herramienta gratuita y en línea que permite una realización anónima por parte de los participantes.
} 
no ha señalado su experiencia docente en la enseñanza pública, pero sí ha aducido que trabaja en el sector privado, en una academia de lenguas. Cuatro profesores afirman no haber tenido ninguna experiencia docente anterior, y los que sí la tienen, afirman haber trabajado dando clases particulares de español e inglés a los alumnos de primaria, impartiendo clases en la universidad o dando clases en academias particulares. Es importante decir que todos los encuestados participan en seminarios de formación.

\subsection{Método de análisis}

Como se ha señalado, debido al escaso número de profesores, se ha elaborado un cuestionario con preguntas abiertas, para que las respuestas obtenidas sean interpretadas de manera cualitativa. Hemos tenido en cuenta la calidad y la diversidad de la enseñanza de ELE previa y los contenidos de los seminarios de formación de profesores.

\section{AnÁlisis y discusión de los Resultados}

\section{Pregunta $n^{\circ}$ 1: Uso de la lengua materna / lengua meta en clase}

Diez encuestados afirman utilizar en clase tanto la lengua materna como la lengua meta, mientras que uno dice emplear solo lengua meta. Es decir, los once participantes usan la lengua meta como lengua de instrucción. Se observa la tendencia de emplear la lengua materna en niveles iniciales y para la explicación de contenidos gramaticales, lo cual nos lleva a la conclusión de que la enseñanza tradicional de lenguas extranjeras sigue estando presente. Según las respuestas, el uso de una lengua o de ambas lenguas varía de una clase a otra, de o una generación a otra, o de un nivel a otro, ya que los profesores quieren asegurarse de que sus alumnos hayan entendido bien los contenidos presentados. Algunos profesores también recurren al lenguaje no verbal (gestos), y uno destaca el uso de materiales audiovisuales, siempre y cuando están disponibles en la escuela donde trabaja.

\section{Pregunta $n^{\circ}$ 2: Conocimientos y competencias que tienden a desarrollar}

En cuanto a los conocimientos y las competencias que tienden a desarrollar, las respuestas muestran una clara tendencia hacia el desarrollo de las cuatro destrezas lingüísticas, con el fin de que los alumnos adquieran competencia comunicativa. Los profesores son conscientes de la importancia de la competencia intercultural, en la que hacen especial hincapié, por lo que trabajan también contenidos culturales e invitan a los alumnos a la reflexión crítica. Puede ser que este interés se deba precisamente a los seminarios de formación que se han realizado en los últimos años (en 2015, por ejemplo, se realizó el seminario bajo el título Desarrollo de la competencia intercultural y uso de las nuevas tecnologías en la enseñanza de ELE). Uno de los participantes $\left(\mathrm{D}^{11}\right)$ ha mostrado estar al día en la legislación sobre los

\footnotetext{
${ }^{11}$ Puesto que se trata de un cuestionario anónimo, hemos marcado las respuestas de los participantes con letras, según el orden en que han contestado.
} 
programas de lenguas extranjeras, lo que interpretamos como un segmento de la formación continua o al menos el interés por esta.

\section{Pregunta $n^{\circ}$ 3: Diferencias individuales entre alumnos}

La tercera pregunta del cuestionario se refiere a diferencias individuales entre alumnos. Los participantes han contestado de forma descriptiva, puesto que les hemos pedido que aduzcan ejemplos que ilustren sus técnicas de trabajo según las diferencias individuales observadas. Las respuestas que prevalecen tienen que ver con el uso de distintas técnicas en la presentación de los contenidos (por ejemplo, vídeo y audio material, lenguaje no verbal, etc.) para que los alumnos, según sus intereses y capacidades, puedan aprender de manera más fácil e interesante. Es decir, de esta forma los profesores motivan a sus alumnos en el proceso de aprendizaje. Varios profesores han expresado explícitamente la interdependencia de las diferencias individuales y la motivación, por lo que con los alumnos más motivados (interesados) trabajan de formas más variadas, mientras que con los alumnos menos motivados (menos interesados) trabajan contenidos básicos, de manera más sencilla, que corresponde a los principios del desarrollo de la competencia lingüística pura. La motivación, según muestran algunas de las respuestas, a veces está relacionada solamente con la nota, por lo que algunos profesores perciben diferencias entre alumnos que estudian para aprender y aquellos que estudian solo para obtener una nota positiva. Una tercera dirección en la que van algunas de las respuestas tiene que ver con la enseñanza inclusiva. Dos profesores han afirmado tener en $\mathrm{su}(\mathrm{s})$ clase(s) alumnos con necesidades educativas especiales, lo que implica una atención individualizada, aplicación de actividades y ejercicios específicos y uso de material complementario. Podemos concluir que todos los profesores reconocen las diferencias individuales y procuran modelar sus clases con el fin de aumentar la motivación de los alumnos y, de ahí, su rendimiento en clase de ELE.

\section{Pregunta $n^{\circ}$ 4: Formas de trabajar}

En cuanto a las formas de trabajar (trabajo individual, en parejas o en grupo), todos los encuestados recurren a las tres formas indicadas, aunque el trabajo en parejas ${ }^{12}$ tiene ligera ventaja en comparación con otros dos, seguido por el trabajo individual. A pesar de que consideran que el trabajo en grupo (de 3 a 5 alumnos) es útil y a la vez presenta un reto, los profesores señalan que es a la vez bastante complicado, porque es difícil mantener la disciplina en clase. Por tanto, el trabajo en parejas, de cierto modo, sustituye el trabajo en grupo, mientras que la actividad individual contribuye, según afirman, al desarrollo de la independencia de los alumnos. Una profesora $(\mathrm{H})$ ha admitido que aplica actividades individuales porque los demás tipos exigen más preparación por parte del profesorado. La respuesta de otra profesora $(\mathrm{K})$ sobre todo ha llamado nuestra atención porque ha descrito de una manera bastante ilustrativa las situaciones a las que aplica cada una de las formas

\footnotetext{
${ }^{12}$ El trabajo realizado en parejas es el más típico del aprendizaje cooperativo de lengua (Richards \& Rodgers, 2009:
} 196). 
y las razones para ello: mientras que con el trabajo individual procura que cada uno de los alumnos se centre en los contenidos y use su propia lógica para entenderlos y trabajarlos, con las actividades en parejas los alumnos pueden consultar, ayudar pero también complementarse uno al otro, cada uno con sus conocimientos; el trabajo en grupo lo usa para unos proyectos más complejos y detallados (por ejemplo, presentar un viaje, una excursión, etc.) así como al final de cada semestre, cuando ya han finalizado las pruebas escritas. La profesora se ha dado cuenta de que a los alumnos les interesa más la nota obtenida que los errores cometidos, por lo que no les gusta hacer la corrección de la prueba, y por esta razón la docente les pide que, bajo su supervisión, vuelvan a hacer la misma prueba, pero no individualmente sino en grupos de cuatro, lo que les permite reflexionar, corregirse unos a otros, enseñarse mutuamente, debatir y, finalmente, comparar las dos versiones de la prueba.

\section{Pregunta $n^{\circ}$ 5: Enseñanza colaborativa (cooperativa)}

En la pregunta número 5 hemos pedido a los profesores que expliquen lo que entienden por enseñanza colaborativa (cooperativa) y que la ilustren con algún ejemplo de su clase. Cinco de los once participantes han dado una explicación de lo que consideran bajo este término, dos han dicho qué es lo que piensan, tres solamente han contestado que la consideran útil, sin más, mientras que una participante ha admitido no conocer este término, pero supone que se trata de enseñanza en grupo, donde cada uno de los alumnos contribuye a la adquisición de los contenidos. Todos los encuestados afirman considerar útil este tipo de enseñanza. Dos profesores han relacionado la enseñanza cooperativa con las nuevas tecnologías y/o internet, tres con distintas fuentes de información y uno piensa que la cooperación tiene que ver con la correlación de distintas asignaturas. A modo de ilustrar la enseñanza cooperativa en sus clases los profesores han dado las siguientes respuestas:

- La búsqueda por parte de alumnos de textos e imágenes que tengan que ver con el tema elegido e investigado.

- Proyectos (en parejas o en grupos) en forma de paneles, presentaciones, exposiciones, etc. con los que se presenta un determinado tema, principalmente cultural, del mundo hispano (flamenco, descubrimiento de América, etc.), lo que permite a los alumnos relacionar los conocimientos de distintas asignaturas, como geografía, historia, música, pero también con las nuevas tecnologías.

- Lluvia de ideas, donde los alumnos, en grupos, dicen el mayor número de palabras que conozcan en relación con el término estudiado.

- Escenificación (y grabación, en algunos casos) de distintas situaciones comunicativas (juegos de roles).

- Debates.

- Aplicación de distintos tipos de WIKIS.

- Concursos que permiten a los alumnos mostrar conocimientos de distintas materias expresadas en español.

De sus respuestas podemos concluir que nuestros profesores aplican la enseñanza cooperativa, aunque no sean conscientes de que se trate de un determinado tipo de instrucción. 
En las actividades que realizan sus alumnos reconocemos algunos de los métodos presentados en la Tabla 1. La heterogeneidad de procedimientos empleados, que varían de una situación a otra, de un grupo a otro, o de un alumno a otro, se corresponde con el aprendizaje colaborativo como término genérico, según han señalado Johnson, Johnson \& Stanne (2000). Los métodos de nuestros profesores también confirman que los alumnos, «mediante el aprendizaje colaborativo aprenden a buscar la información, a compartirla, a comunicarse y a autoevaluarse de forma colaborativa» (Carrió Pastor, 2007: 2), mientras que el profesor tiene un papel secundario, periférico y sirve, como se ha dicho, de facilitador.

\section{Pregunta $n^{\circ}$ 6: Uso de las nuevas tecnologías y redes sociales}

En lo referente al uso de las nuevas tecnologías y redes sociales como soporte en la realización de algunas actividades, en clase y fuera de ella, las respuestas han mostrado que todos los profesores utilizan o incitan a los alumnos a utilizar las nuevas tecnologías, mientras que las redes sociales no son utilizadas aún. Además de usar los ordenadores para proyectar películas y audio materiales, los profesores también animan a sus alumnos a hacer vídeos cortos o clips, para presentar diferentes temas o situaciones comunicativas. Uno de los participantes (A) hasta organiza visitas virtuales a diferentes museos, pero también motiva a los alumnos a ampliar el vocabulario mediante la búsqueda adecuada en internet. Asimismo, observamos una doble presencia o una doble interpretación de la función de las nuevas tecnologías en la enseñanza: por un lado, facilitan la adquisición de lengua extranjera, y por otro, sirven para concienciar a los alumnos sobre el uso correcto de las nuevas tecnologías e internet y cómo sacarle el mayor partido (por ejemplo, recomendación de las páginas web, el uso de palabras clave en búsqueda de determinados temas, aplicaciones, etc.).

\section{Pregunta $n^{\circ}$ 7: Alumnos como colaboradores activos en clase}

Los once profesores encuestados afirman que sus alumnos son participantes o colaboradores activos en el proceso del aprendizaje. Resumimos a continuación los ejemplos de su aprendizaje cooperativo:

- Alumnos como ayudantes del profesor y como enseñantes.

- Importancia de la opinión de los alumnos en la elección de los contenidos y las formas de trabajo en clase; asimismo, los alumnos tienen la libertad y el derecho a decir si algunos contenidos y las formas de trabajar les gustan o no y por qué.

- Mutua ayuda de los alumnos en el proceso de aprendizaje.

- Explotación en clase de actividades, intereses y habilidades de los alumnos extraescolares (por ejemplo, los alumnos que tienen una formación musical tocan o cantan canciones en español, etc.).

- Preparación y participación en los concursos y juegos de roles.

Consideramos importante señalar que varios profesores han subrayado la importancia de un ambiente relajado y agradable en clase, con el que se incentiva a los alumnos a trabajar e interactuar. Todos estos ejemplos confirman los postulados de Jokikokko (2005: 76), que 
habla de una pedagogía transformacional, cuyos elementos principales son la creación de la confianza en el aula y el establecimiento del diálogo entre los alumnos y el profesor.

\section{Pregunta $n^{\circ}$ 8: Trayectoria docente}

Finalmente, hemos preguntado a los profesores si han cambiado algo a lo largo de su carrera docente, de qué manera y por qué. Las respuestas obtenidas muestran indudablemente que las nuevas tecnologías han ganado terreno en clase de ELE. Los profesores ven en las nuevas tecnologías una fuente de motivación para los alumnos, puesto que se trata de medios que los alumnos utilizan a diario y conocen muy bien, lo cual contribuye directamente a que las clases sean más prácticas, o sea, permite a los alumnos un uso real de la lengua. Como dice la profesora F, «los niños de hoy son más visuales y reaccionan y aprenden mejor con los estímulos en forma de nuevas tecnologías». De igual manera, está claro que los profesores utilizan menos el método frontal y trabajan más en el desarrollo de la competencia comunicativa (e) intercultural, mediante el uso de los medios audiovisuales, a pesar de las dificultades (si no carencias) técnicas con las que se encuentran a menudo. Según afirma la profesora K, el método frontal, tradicional «mata la creatividad, la curiosidad y la individualidad, porque los profesores ya no son la única fuente de conocimientos, sino que están ahí para orientar a los alumnos, ayudarles a encontrar respuestas». Actitudes como esta muestran un cambio importante en comparación con previos estudios realizados por Pejović y Jovanović (2012) y Jovanović y Pejović (2013), que mostraron la supremacía del enfoque gramatical y léxico. Según Pejović y Jovanović (2012: 163), la prevalencia del enfoque gramatical se debía posiblemente «a la experiencia previa de las participantes quienes, a lo largo de su educación primaria y secundaria (en muchos casos), recibieron una enseñanza tradicional, que ha influido en la manera de percibir el proceso educativo y de formar modelo de la enseñanza de lenguas extranjeras». En las Estrategias de la educación en Serbia hasta 2020, elaboradas por el Ministerio de Educación y Ciencia de la República de Serbia en 2012, se hace constar que los nuevos (modernos) métodos todavía no se aplican en medida suficiente, debido a que proceden de una distinta percepción del método de enseñanza y del papel del profesor y del alumno. Podríamos decir que esto confirma algunos de los resultados obtenidos por Johnson, Johnson \& Stanne (2000: 13), que afirman que existen dos aproximaciones a la implementación del aprendizaje colaborativo: directo y conceptual. Los partidarios del enfoque directo consideran que los profesores pueden aprender y aplicar en poco tiempo las técnicas específicas de este tipo de aprendizaje, mientras que el enfoque conceptual aboga por el aprendizaje del sistema conceptual por parte de profesores. De este modo no solo sería posible la adaptación de las clases y las actividades, sino que los resultados serían duraderos. Como se ha indicado, sin embargo, las respuestas que hemos obtenido de nuestros participantes muestran que sí hay un claro avance al respecto, lo que atribuimos a dos factores: seminarios de formación de profesores y la realidad misma, o sea, las necesidades y las características de los alumnos de hoy, que viven en la era digital. La profesora $\mathrm{C}$ ha señalado la importancia del grupo con el que trabaja, porque dependiendo del grupo elige los métodos y las técnicas, lo que demuestra que la profesora es muy consciente de diferencias individuales. En las Estrategias también llama la atención el tamaño de los grupos en clase: debido a los recortes en la enseñanza, el número de clases ha disminuido a favor del número de alumnos por clase, 
lo que dificulta significativamente el proceso educativo en general, y de lenguas extranjeras en particular. Y si se añade que el número de horas semanales de lengua extranjera es de dos (de 45 minutos), es evidente que los profesores no tienen una tarea fácil. Una respuesta más ha llamado nuestra atención: una de las participantes $(\mathrm{J})$ ha subrayado la importancia de seminarios de formación de profesorado, que en su caso la han inspirado y le han dado «nuevas ideas» para trabajar. El intercambio de experiencia con los demás profesores durante los seminarios también le ha parecido sumamente útil e importante. Esta actitud positiva hacia los seminarios y la interacción con los colegas muestra que en un período bastante breve, desde un primer estudio de este tipo (Pejović y Jovanović, 2012; Jovanović y Pejović, 2013) se ha avanzado también en la organización de los seminarios de formación continua, que son cada vez más prácticos y concretos. ${ }^{13}$

\section{Pregunta $n^{\circ}$ 9: Mejora de la labor docente}

Con la última pregunta hemos querido saber si los profesores consideran necesario y están dispuestos a mejorar algunos aspectos de su labor docente y de qué manera. La mayoría aboga por un mayor uso de las nuevas tecnologías en clase y por el desarrollo de la competencia digital. No obstante, aparte de que las condiciones técnicas no son óptimas, el programa de estudio, según los profesores, no está en correlación con el número de clases semanales. (En las Estrategias se reclama lo mismo.) Y, cuando a todo ello se añaden las pruebas escritas y orales, no queda suficiente espacio no solo para el uso de las nuevas tecnologías y los nuevos métodos en clase, sino que la calidad de la enseñanza misma disminuye, junto con la motivación de los alumnos. A pesar de ello los profesores expresan su deseo de formarse continuamente, a través de los seminarios, intercambios de experiencias y ejemplos de buenas prácticas, con lo que muestran un alto grado de responsabilidad y conciencia vocacional.

\section{Conclusiones}

Los resultados de la investigación que hemos llevado a cabo muestran que los profesores de ELE en la República de Serbia por lo general siguen las nuevas tendencias en la didáctica de lenguas extranjeras. En comparación con los resultados de estudios anteriores, que revelaron una tendencia clara hacia el enfoque netamente lingüístico, las respuestas obtenidas muestran un evidente avance en el empleo del enfoque comunicativo en clase, y dentro de él, en el uso de una enseñanza interactiva y colaborativa. Aunque no siempre sean conscientes de ello, los profesores recurren al aprendizaje cooperativo en sus clases, lo cual corrobora el hecho de que el trabajo en parejas y en grupo predomine sobre el trabajo individual, igual que lo confirma la heterogeneidad de las técnicas y actividades elegidas

\footnotetext{
${ }^{13}$ Pejović \& Jovanović (2012: 162-163) señalaron la necesidad de seminario prácticos: «(...) las participantes demuestran gran inclinación hacia la mejora de sus estilos de enseñanza. (...) De los resultados obtenidos en la encuesta se desprende que los profesores serbios de español necesitan formación. Deberían ser además cursos prácticos, ofrecer múltiples situaciones y soluciones a posibles problemas. (...) no es suficiente que los profesores conozcan las teorías más modernas en la enseñanza de lenguas extranjeras, sino que es necesario crear condiciones para que ellos mismos cuestionen sus ideas y actitudes sobre la enseñanza, y para que tengan la oportunidad de conocer de manera directa diferentes enfoques».
} 
según los intereses de los alumnos. Además, los profesores manifiestan un alto grado de responsabilidad y una actitud crítica hacia la enseñanza de ELE en general y hacia su propio estilo de enseñanza. Igualmente, recurriendo al autoanálisis y al meta-análisis de las técnicas instructivas que emplean en clase, en búsqueda de estrategias y métodos óptimos, los profesores muestran un evidente afán de motivar a los alumnos, capacitarlos para ser autónomos a largo plazo, para poder encontrar la información que necesiten y para producir textos escritos $\mathrm{u}$ orales adecuados.

También se ha mostrado que los profesores de español serbios tienen una actitud positiva hacia los seminarios de formación y que aplican en clase los conocimientos adquiridos. De ahí queda patente la necesidad de seguir organizándolos, ofreciendo actividades concretas y eficaces que los profesores puedan aplicar en clase y con las que puedan enseñar a los alumnos determinadas estrategias de aprendizaje a partir de las que podrán desarrollar otras nuevas, sus propias estrategias. Puesto que los profesores recurren a la enseñanza colaborativa / cooperativa de manera no del todo consciente, se desprende que en los seminarios habría que ofrecerles más información, tanto teórica como práctica, sobre este tipo de instrucción. Finalmente, el tema de futuros seminarios y futuras investigaciones tendría que ser la evaluación de los métodos del aprendizaje cooperativo.

\section{REFERENCIAS}

Branković, D. \& Mikanović, B. (2011). "Emancipatorsko vaspitanje - osnov razvoja odnosa u nastavi", in Pedagoška stvarnost, 7-8: 581-595.

Carrió Pastor, M.L. (2007). "Ventajas del uso de la tecnología en el aprendizaje colaborativo ", in Revista Iberoamericana de Educación, 41/4. Available from: http://rieoei.org/1640.htm, accessed 9 March, 2017.

Dillenbourg P. (1999). "What do you mean by collaborative learning?", in P. Dillenbourg (ed.), Collaborative-learning: Cognitive and Computational Approaches. Oxford: Elsevier, 1-19.

Freire, P. (1998). Pedagogy of Freedom: Ethics, Democracy, and Civic Courage, Lanham: Rowman \& Littlefield Publishers, Inc.

Johnson, D.W., Johnson, R.T. \& Stanne, M.B. (2000.). "Cooperative Learning methods: A MetaAnalysis". Cooperative Learning Center at the University of Minnesota. Available from: https://pdfs.semanticscholar.org/93e9/97fd0e883cf7cceb3b1b612096c27aa40f90.pdf accessed 25 March, 2017.

Jokikokko, J. (2005). "Interculturally trained Finnish teachers' conceptions of diversity and intercultural competence", in Intercultural Education, 16: 69-83.

Jovanović, A. \& Pejović, A. (2012). "Kontinuirano obrazovanje nastavnika stranog jezika: potrebe i izazovi“, in S. Gudurić (ed.), Jezici i kulture u vremenu i prostoru (Zbornik radova). Novi Sad: Filozofski fakultet, 407-416.

Jovanović, A. \& Sánchez Radulović, N. (2013). "El español en Serbia: estado de la cuestión", in Colindancias, 4: 373-392.

Mikanović, B. (2015). "Humanističko vaspitanje i emancipacija ličnosti", in Humanistički ideali obrazovanja, vaspitanja i psihologije (Tematski zbornik radova). Niš: Filozofski fakultet Univerziteta u Nišu, 223-235.

Panitz, T. (1997). "Collaborative versus cooperative learning - A comparison of the two concepts which will help us understand the underlying nature of interactive learning", in Cooperative Learning and College Teaching, 8 (2). Available from: http://files.eric.ed.gov/fulltext/ ED448443.pdf, accessed 7 April, 2017. 
Pejović, A. \& Jovanović, A. (2012). "Actitudes epistemológicas de los profesores de español como lengua extranjera", in Colindancias, 3: 157-163.

Richards, J. C. \& Rodgers, T. S. (2003). Enfoques y métodos en la enseñanza de idiomas. Madrid: Edinumen.

Sharan, Y. (2014). "Learning to cooperate for cooperative learning", in Anales de psicología, vol. $30, \mathrm{n}^{\circ} 3: 802-807$.

Strategije razvoja obrazovanja u RS do 2020. godine Available from: http://www.mpn.gov.rs/wpcontent/uploads/2015/08/STRATEGIJA-OBRAZOVANJA.pdf, accessed 25 March, 2017.

Trujillo Sáez, F. \& Ariza Pérez, M.A. (Eds.) (2006). Experiencias Educativas en Aprendizaje Cooperativo. Available from: http://fernandotrujillo.es/wp-content/uploads/2010/05/AC_libro. pdf, accessed 19 March, 2017.

Trujillo Sáez, F. 2002. "Aprendizaje cooperativo para la enseñanza de lengua“, in Publicaciones, 32: 147-162. 